\title{
Temporal Analysis of the Antibody Response to HIV Envelope Protein in HIV-infected Laboratory Workers
}

Seth H. Pincus, Katherine G. Messer, Peter L. Nara, " William A. Blattner, " Ginga Colclough, ${ }^{\star}$ and Marvin Reitz Laboratory of Microbial Structure and Function, Rocky Mountain Laboratories, National Institute of Allergy and Infectious Disease, Hamilton, Montana 59840; * National Cancer Institute, Bethesda, Maryland 20892, and Cancer Research and Development Center, Frederick, Maryland 21702; and ${ }^{\ddagger}$ Research Triangle Institute, Rockville, Maryland 20852

\begin{abstract}
Three laboratory workers have been infected with the IIIB strain of HIV; their antibody response to HIV has been studied in serial serum specimens. Because the infecting virus is known, the fine specificity of the antibody response was studied on the homologous strain of HIV. Anti-p17, anti-p24, antigp160, CD4/gp1 20 blocking and neutralizing antibodies developed in parallel. Epitope mapping of the anti-gp160 response indicated several regions that consistently induced an antibody response. Serum contained antibody which reacted with V3-specific peptides corresponding to the very tip of the loop and crossreactivity was seen with V3 loop peptides from other sequence divergent strains of HIV. Antibody to the V1 loop was produced at levels comparable with that seen for the V3-loop. Anti-V1 neutralized HIV with a titration curve equivalent to an anti-V3 monoclonal antibody. Because the infecting virus is known and serial reisolates have been obtained, we explored the relationship between production of antibody to a given epitope and mutation in the virus. The data suggest that an association exists, but do not clearly indicate that antibody drives the selection for mutant viruses. The findings presented here provide a fine specificity analysis of the evolution of the antibody response to $\mathrm{HIV}$ in greater detail than has previously been performed. (J. Clin. Invest. 1994. 93:2505-2513.) Key words: HIV • AIDS • antibody
\end{abstract}

\section{Introduction}

Evaluation of the immune response to HIV is complicated by sequence variability of viral proteins, particularly of the envelope protein(s) gp160. The infecting isolate of HIV is rarely known and the immune response is frequently measured on recombinant proteins, peptides, and virus isolates that correspond to frequently used laboratory strains of $\operatorname{HIV}(1,2)$. Thus, the human immune response to the infecting strain of HIV has not been fully assessed. Because important antigenic structures may be localized in the variable regions of the viral proteins, it is possible that significant aspects of the response may be missed. In previous studies we have epitope mapped the systemic antibody response in three sets of subjects, all of whom have been exposed to gp 160 derived from a single strain of HIV (IIIB/LAV) $(3,4)$. The subjects include humans im-

Address correspondence to Dr. Seth Pincus, Laboratory of Microbial Structure and Function, Rocky Mountain Laboratories, Hamilton, MT 59840.

Received for publication 29 October 1993 and in revised form 7 February 1994

The Journal of Clinical Investigation, Inc.

Volume 93, June 1994, 2505-2513 munized with gp160 subunit vaccines, chimpanzees immunized with gp 160 and then challenged with live HIV, and laboratory workers accidently infected with HIV during the course of their experiments (5). In this communication, we report on the temporal evolution of the antibody response to gp160 in the infected laboratory workers. The data are consistent with the premise that testing for antibody responses on homologous HIV may reveal important epitopes that may be missed when heterologous strains are used.

\section{Methods}

Infected laboratory workers. Three laboratory workers, all of whom worked with highly concentrated HIV, have been infected with the IIIB/LAV strain of HIV during the course of their work. The first reported worker, L.W.F., has been described elsewhere (5). The event causing infection occurred in 1985 and has not been defined. L.W.F. is currently healthy and stable, with a CD4 cell count $<500 / \mathrm{mm}^{3}$. Treatment with vaccine therapy (6) began in February 1993. L.W.R. was infected in 1985 possibly because of a skin puncture with a chemically decontaminated elutriator needle. L.W.R. seroconverted in late 1985 and developed AIDS in May 1991. He has received AZT since June 1991, ddI since March 1992, and vaccine therapy was instituted in December 1992. L.W.S. was infected in 1990 after a heavy exposure of skin and mucous membranes to highly concentrated HIV. L.W.S. is currently healthy and clinically stable with a CD4 cell count consistently $<300 / \mathrm{mm}^{3}$. Therapy with AZT began in 1990, ddI in 1992, and vaccine therapy was added in December 1992. Serial serum samples were obtained on the lab workers from the earliest time point available until December 1992. Since vaccine therapy started in December 1992 or February 1993, any later time points will not reflect the natural evolution of the antibody response. Sequences of the complete envelope gene from the laboratory workers were obtained either by viral isolation and molecular cloning of the provirus or by PCR from peripheral blood leukocytes. ${ }^{1}$ Sequence analysis has confirmed that the laboratory workers were infected with the IIIB/LAV strain.

Immunoassays. All assays used in this study are described in detail elsewhere (3). Unless otherwise noted, all recombinant proteins and peptides correspond to the IIIB/LAV strain of HIV. Sera were tested in ELISA at a 1:1,000 dilution. Microtiter wells were coated with recombinant proteins at $1 \mu \mathrm{g} / \mathrm{ml}$ and soluble peptides at $5 \mu \mathrm{g} / \mathrm{ml}$. The following recombinant antigens were used: baculoviral gp 160 ${ }_{\text {IIIB }}$ (Repligen, Cambridge, MA), gp 120 ${ }_{\text {IIIB }}$ (American Bio-Technologies, Cambridge, MA), p24 (National Institutes of Health AIDS Research and Reference Reagent Program, Rockville, MD), and p17 (MRC AIDS Reagent Project, Potters Bar, UK). Synthetic peptides were synthesized using a peptide synthesizer (model 431 A; Applied Biosystems Inc., Foster City, CA). The following synthetic peptides were used: $\mathrm{V} 1_{\text {IIIB }}$ (amino acids [AA] ${ }^{2}$ 131-156, numbering according to reference 7 , sequence of HXB-2B) CTDLKNDTNTNSSSGRMIMEKGEIKNC,

1. Reitz, M. S., L. Hall, M. Robert-Guroff, J. Lautenberger, B. Hahn, G. Shaw, L. Kong, S. Weiss, D. Waters, R. C. Gallo, and W. A. Blattner, manuscript submitted for publication.

2. Abbreviations used in this paper: AA, amino acid; s, soluble. 
V3 $_{\text {IIIB }}$ (AA 297-330) TRPNNNTRKSIRIQRGPGRAFVTIGKIGNMRQAH, and mid-gp41 ${ }_{\text {IIIB }}$ (AA 718-743) QTHLPTPRGPDRPEGIEEEGGERDRD. Peptides corresponding to the V3 loop of the HIV strains MN and RF were purchased from American Bio-Technologies. The sequences of the peptides were as follows: $\mathrm{MN}$ loop, CNKRKRIHIGPGRAFYTTKN; RF loop, CNTRKSITKGPGRVIYATGQ. Antibodies were detected with alkaline phosphatase-conjugated anti-human immunoglobulins (anti-IgG, I-A, and I-M; Zymed Laboratories, South San Francisco, CA) and $p$-nitrophenyl phosphate substrate (Sigma Chemical Co., St. Louis, MO). Data are reported as $\mathrm{A}_{405}$ and represent means of triplicate values.

Epitope scanning was performed by the method of Geysen (8). A set of 283 overlapping 12-mer peptides was constructed on solid supports using materials and protocols supplied by Cambridge Research Biochemicals (Wilmington, DE). Peptides began every third AA (starting at the amino terminus) and thus had a nine-AA overlap. Sequences used to construct the peptides conform to those of the BH10 isolate of HTLV-IIIB. Three control pins with irrelevant peptides were included. Before use, the peptides were blocked with 2\% BSA (Sigma Chemical Co.), $0.1 \%$ Tween 20 in PBS. The supports were incubated with the test serum diluted 1:1,000 in PBS. 2\% BSA, followed by successive washings and incubation with alkaline phosphatase-conjugated goat anti-human Ig antibody and then substrate. Data are reported with the background absorbance of the control pins subtracted. After each use of the peptide support matrix, antibodies were rigorously desorbed, and the pins tested for residual antibody ( 3 ).

Inhibition of the interaction between soluble CD4 (sCD4) and gp120 (American Bio-Technologies) was measured by ELISA, using immobilized gp120 and biotin-labeled sCD4. Sera to be tested for blocking antibodies were first added to the immobilized gp 120. BiotinsCD4 was added $2 \mathrm{~h}$ later, and the mixture was incubated overnight. Binding of SCD4 was detected with alkaline phosphatase-conjugated avidin and substrate. The specificity of the assay was demonstrated with appropriate monoclonal antibodies (3).
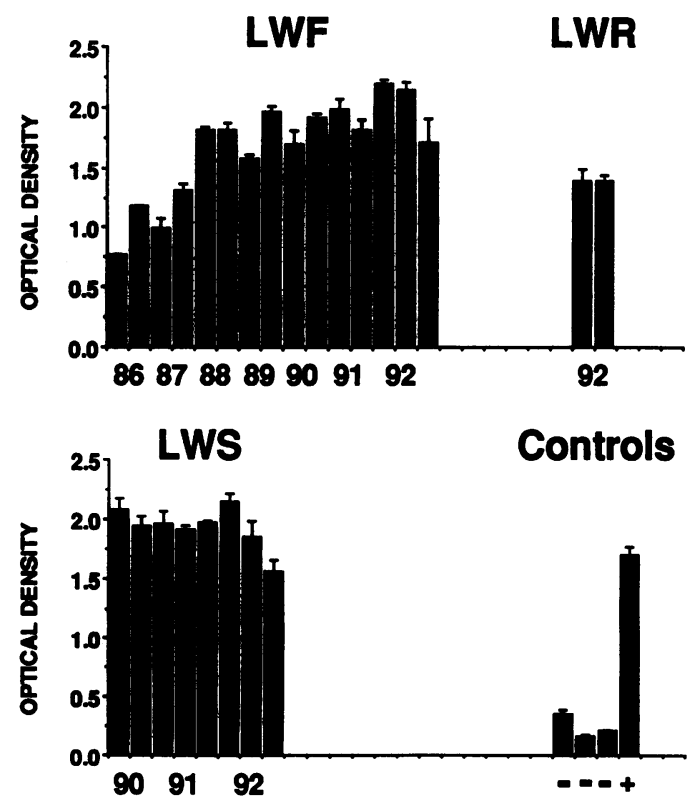

Figure 1. Anti-gp160 antibody response. Anti-gp160 antibodies were measured by ELISA on rgp 160. Serial serum samples from each laboratory worker were tested at a 1:1,000 dilution. Antibody binding was detected with alkaline phosphatase goat anti-human Ig and the colorimetric substrate $p$-nitrophenyl phosphate. $\mathrm{A}_{405}$ is shown on the vertical axis. The specimens are arranged serially on the horizontal axis, with the years labeled. Data are the mean and SE of triplicate samples. Negative controls are normal human sera; the positive control is pooled HIV immune globulin.
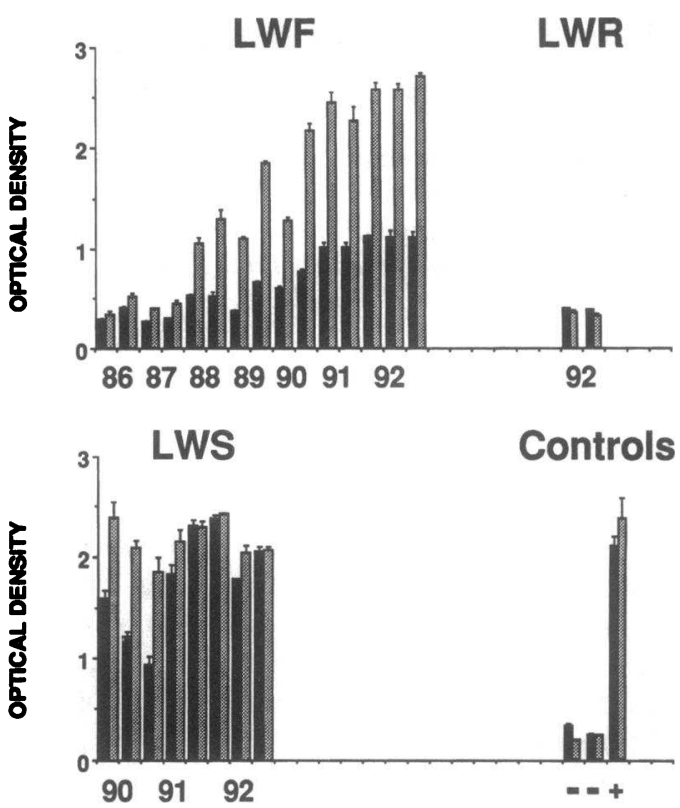

Figure 2. Anti-Gag antibody response. Anti-p17 (black bars) and anti-p24 (dotted bars) antibody levels were determined by ELISA. Conditions were as described for Fig. 1.

Neutralization assays. Virus neutralization was performed using a focal infectivity assay $(3,9-11)$. The molecularly cloned HIV strains NL4-3 (12) and MN (13) were used in the neutralization assays. Env of NL4-3 is derived from LAV. A pretitered dilution of cell-free virus was mixed with the serum to be tested and incubated for $1.5 \mathrm{~h}$ at $37^{\circ} \mathrm{C}$. The mixture was then transferred to a monolayer of CD4+ HeLa cells (cell line 1022) for $3 \mathrm{~h}$. The monolayer was washed free of virus and antibody, seeded with fresh media, and incubated for $3 \mathrm{~d}$. The cells were fixed, and HIV-infected cells were identified by immunoperoxidase staining associated with the characteristic morphology of a syncytium. Neutralizations on serial viral isolates from L.W.F. were performed by a quantitative microtiter syncytium-forming assay using CEM-SS cells (14). Briefly, 50,000 cells to be infected were attached to poly-L-lysinecoated microtiter wells. HIV-1 stocks were used at a concentration of 100-250 syncytium-forming units and mixed with the test sera at twofold serial dilutions. The cells were incubated with the antibody-virus mixture for $1 \mathrm{~h}$ at room temperature after which they were washed and replaced in complete RPMI 1640 . Syncytia corresponding to a single focus of infection were counted on day 5 and compared with values in control wells (normal human serum). The endpoint neutralization titer was defined as the reciprocal of the highest antibody dilution giving $90 \%$ inhibition of syncytia formation.

Affinity purification of anti-V1 antibody. V1 loop peptide was immobilized on agarose (SulfoLink Gel; Pierce, Rockford, IL) through the terminal cysteine residues at $2.4 \mathrm{mg}$ peptide $/ 1.5 \mathrm{ml}$ of gel. Aliquots ( $0.5 \mathrm{ml}$ ) of L.W.F. serum (June 1991) were passed over the column, and the column was washed with PBS and then $0.5 \mathrm{M} \mathrm{NaCl}, 0.01 \mathrm{M}$ EDTA. The purified antibody was desorbed with $0.2 \mathrm{M}$ glycine, $\mathrm{pH}$ 2.5. No additional antibody was desorbed with $6 \mathrm{M}$ guanidine $\mathrm{HCl}$. Desorbed antibody was neutralized immediately and dialyzed against PBS. As a control, normal serum was subjected to the identical procedure and concentrated to the same final volume.

\section{Results}

Antibody to Env and Gag proteins. Serum anti-Env antibody was measured by ELISA on recombinant gp160 (Fig. 1). Antibody levels in L.W.F. slowly rose from 1986 until 1989 and then remained reasonably constant. It is not possible to state 
LWF 8/86

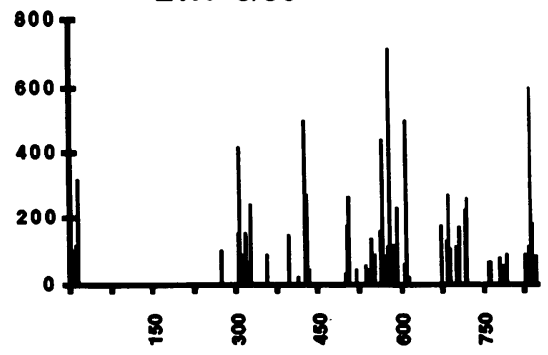

LWF 9/90

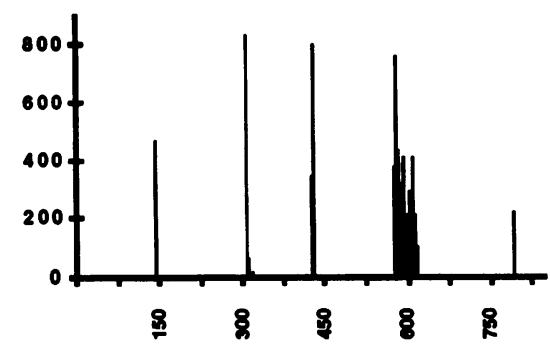

LWF 7/92

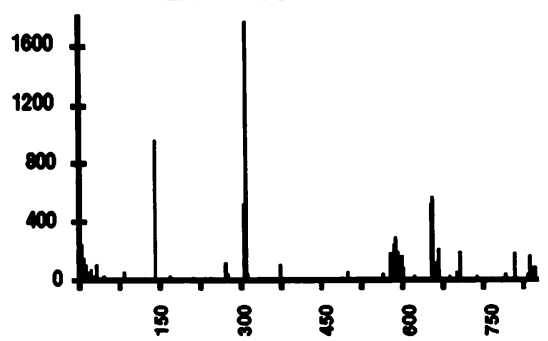

LWS 9/90

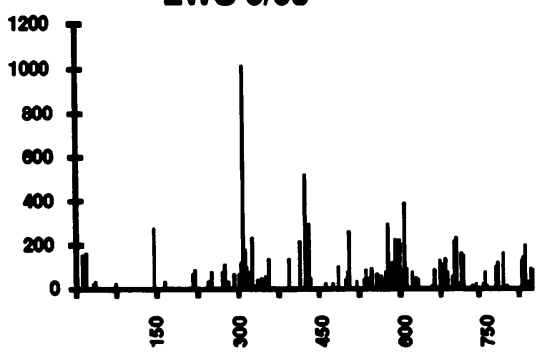

LWS 8/92

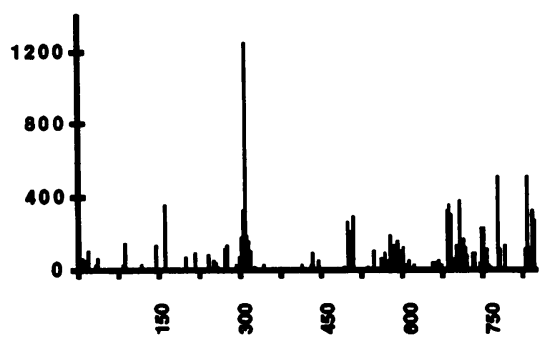

LWR 11/92

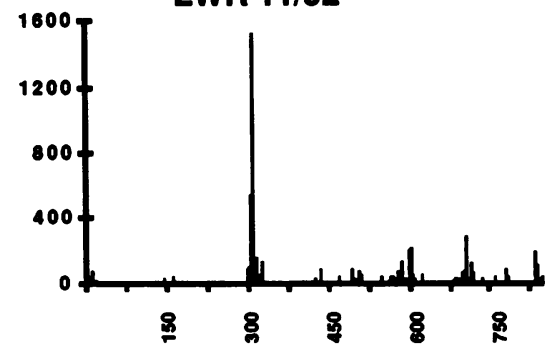

NHS

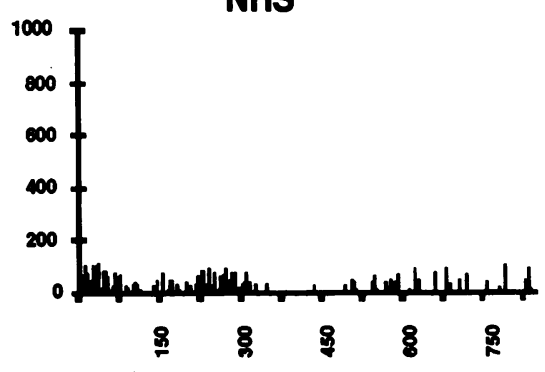

HIVIG
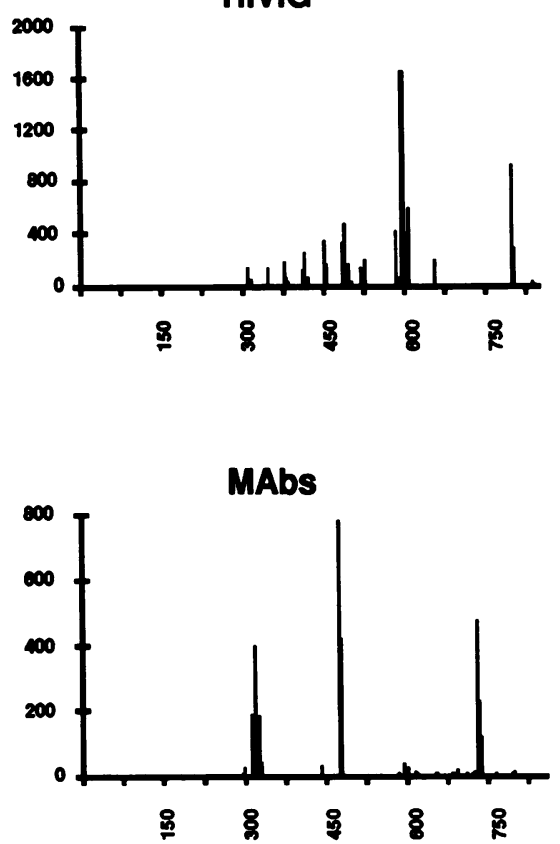

Figure 3. Peptide scanning of laboratory worker sera. All laboratory worker sera were tested for binding to a set of 283 overlapping 12 -mer Geysen peptides representing the entire sequence of the IIIB/LAV Env protein. Six analyses representing early and late time points are shown. Controls include a normal serum, a pooled preparation of HIV immune globulin, and three monoclonal antibodies recognizing linear epitopes. The numbers on the horizontal axis indicate the numerical position of the first amino acid of each peptide. The peptides attached to the supports are arrayed on the horizontal axis with the closest to the amino terminus on the left and proceeding toward the carboxyl-terminal peptides to the right. Binding of antibody to each peptide is shown on the vertical axis as $\mathrm{A}_{405} \times 10^{3}$ (minus binding to control pins with no peptide). Because each sample was run on a different day, the level of binding seen in one sample cannot be compared with that seen in another.

whether the last value represents the beginning of a true fall in the antibody level, since vaccine therapy was initiated shortly thereafter. L.W.S. has demonstrated consistently high levels of antibody from the initial time point. Antibody levels in L.W.S. appeared to be falling through 1992 . Only two serum samples were available from L.W.R., both obtained while the patient had AIDS. Both of these samples had less antibody than was seen at plateau levels in the other two laboratory workers. At all time points, antibody levels in all three patients were significantly higher than those seen in seronegative controls. Although antibody levels shown in Fig. 1 were from a single 1:1,000 dilution, we have performed titration curves, and these results reflect those seen with titrations.

Antibody to the core proteins p17 and p24 are shown in Fig. 2. As with antibody to gp160, levels in L.W.F. rose and then levelled off, while those in L.W.S. started at a high level and remained high through the entire time course. Initial levels of anti-Gag in L.W.F. were not significantly higher than seen in seronegative controls. We failed to detect anti-Gag in L.W.R.
Fine specificity of anti-Env. The fine specificity of the antibody response to gp 160 was measured using synthetic peptide antigens in two different formats: soluble peptides used to coat microtiter wells and Geysen peptides immobilized on solid supports $(3,4,8)$. The latter are best used to localize antigenic regions because large numbers of overlapping peptides can be synthesized. But this method is semiquantitative at best, since only a single serum was tested at a time. To quantitate antibody levels to important epitopes, soluble peptides were used.

We have identified the immunogenic regions of gp160 in the three laboratory workers at all time points using a set of 283 overlapping 12-mer Geysen peptides. Although there were differences between the laboratory workers and at different times in individuals, there were several regions of gp160 that were consistently immunogenic. Representative analyses are shown in Fig. 3. Immunogenic regions of gp120 included the $\mathrm{NH}_{2}$ terminus (AA 1-20), V1 loop (AA 142-153), V3 loop (AA 300-330), C5 domain (AA 425-450), and the COOH terminus (AA 480-510). In gp41, the "immunodominant" re- 
gion (AA 580-610) and the $\mathrm{COOH}$ terminus (AA 820-850) bound antibody.

Because antibody to the V1 and V3 loops was seen in most peptide scanning analyses of the laboratory worker sera, soluble peptides corresponding to these regions were constructed. ELISA was performed with both of these peptides in the same experiment, and the results are shown in Fig. 4. In most serum samples, with the exception of the early L.W.S. specimens, there were equivalent levels of antibody to the V1 and the V3 loops. This was surprising, since the V3 loop has been identified as a major immunodominant domain $(1,15)$, while the V1 loop has not previously been shown to be antigenic. Antibody levels to the $\mathrm{V} 3$ loop showed strikingly different temporal patterns in L.W.F. and L.W.S., with a rising trend seen in L.W.F. and a decline in L.W.S. with time. L.W.R. made low levels of antibody to the V3 loop.

The fine specificity of the anti-V 3 response has been tested in two ways: mapping on overlapping 12-mer Geysen peptides and testing for crossreactivity on V3 loop peptides corresponding to other strains of HIV. Mapping on the overlapping peptides demonstrated that every serum sample preferentially bound the peptide that corresponded with the tip of the loop (Fig. 5). Although there was reactivity seen with peptides corresponding to other portions of the loop, the greatest reactivity was to the tip, which contains the well conserved core sequence GPGR (16). Crossreactivity of anti-V3 antibodies was assessed on peptides corresponding to the V3 loops of the strains $\mathrm{MN}$ and RF (Fig. 6). Different patterns of crossreaction were seen among the laboratory workers. L.W.F. recognized the RF loop, particularly in the later serum samples, while neither L.W.R. nor L.W.S. sera bound this peptide. All lab workers showed considerable reactivity with the MN loop peptide. The positive control, a pool of immune globulins from
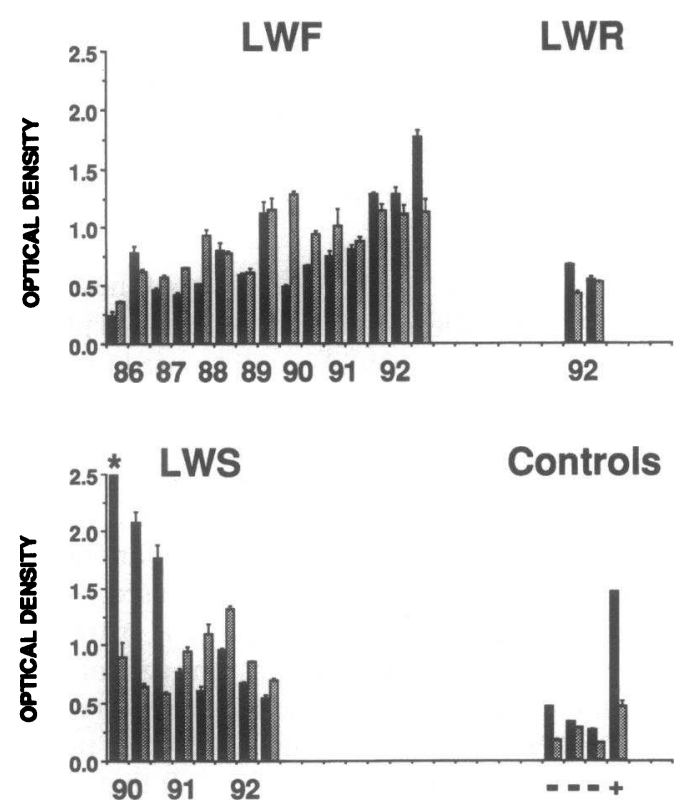

Figure 4. Binding of antibody to peptides representing V3 (black bars) and V1 (dotted bars) loops. Antibodies to the V1 and V3 loops were detected by ELISA on peptides corresponding to sequences of these regions. Conditions were as described for Fig. 1. seropositive patients, preferentially reacts with $\mathrm{MN}$ and $\mathrm{RF}$ peptides.

Because we have reported previously that immunization with vaccinia-gp 160 primes both humans and chimpanzees to respond to an epitope in mid-gp41 (AA 720-740) upon subsequent exposure to gp160 in the form of either recombinant protein or live HIV $(3,4)$, we have analyzed the LW sera for reactivity to this epitope. None of the sera bound to a peptide corresponding to this region of gp41 (data not shown).

Assays of functional antibody activity. The ability of antibody to block the interaction of CD4 with gp120 is an important component of neutralizing antibody $(2,17-19)$. Using immobilized gp120 and biotinylated sCD4, we have analyzed the ability of the laboratory worker sera to block this interaction (Fig. 7). Both L.W.F. and L.W.S. showed temporal patterns of this antibody similar to that seen with all their other antibody responses, i.e., L.W.F. showed increasing amounts of antibody with time, whereas L.W.S. had high levels from the beginning and maintained these levels. Interestingly L.W.R., who produced only low levels of antibody to most other HIV antigens, had high levels of gp120/CD4 blocking antibody. Although the CD4-binding site on gp120 is thought to be a conformational structure involving multiple regions of gp 120 $(17,20)$, one region in particular (AA 425-440) has been implicated as being particularly important $(21,22)$. We have constructed a peptide corresponding to this region (AA 418-441) and measured antibody binding. There was no correlation between the presence of antibody binding to this peptide and inhibition of gp120/CD4 binding (data not shown).

We have measured virus neutralization using a focal infectivity assay $(3,9-11)$. Neutralization was measured on homologous (NL4-3) and heterologous (MN) strains of HIV. Fig. 8 shows the results obtained on NL4-3. L.W.F. showed increasing titers of neutralizing antibody with time, L.W.S. peaked in 1991, and L.W.R. failed to demonstrate any neutralization. Similar patterns of neutralization were seen with MN (data not shown).

Because there was a significant antibody response to the V1 loop, we have asked whether this antibody has any neutralizing activity. We have affinity-purified anti-V1 antibody from L.W.F. on immobilized V1 peptide. We have confirmed that this antibody reacts only with the V1 loop as follows: $(a)$ it reacts only with V1 peptide and no other Geysen or soluble peptide used in this study, and $(b)$ it binds to a recombinant gp120 construct, but not an identical construct lacking the V1 and V2 loops. Fig. 9 shows titration curves of neutralization obtained with affinity-purified anti-V1 and an anti-V3 monoclonal antibody on the HIV strain NL4-3. The results show that the anti-V1 has neutralization activity equivalent to antiV3. No neutralization was seen with a normal human serum purified in a fashion similar to the anti-V1, with purified normal human immunoglobulins at the same concentration, nor of a murine leukemia virus with the anti-V1 antibody.

Analysis of relationship between epitopes and mutations. Sequences of Env from L.W.F. have been obtained at multiple time points during the course of infection. ${ }^{1} 10$ sequences of the complete envelope gene were obtained from 1985 to 1992. During this time there were 18 mutations from the initial sequence that were seen at least two different times. To determine whether there was a relationship between the presence of antibody to a given region of gp160 and a mutation in the 

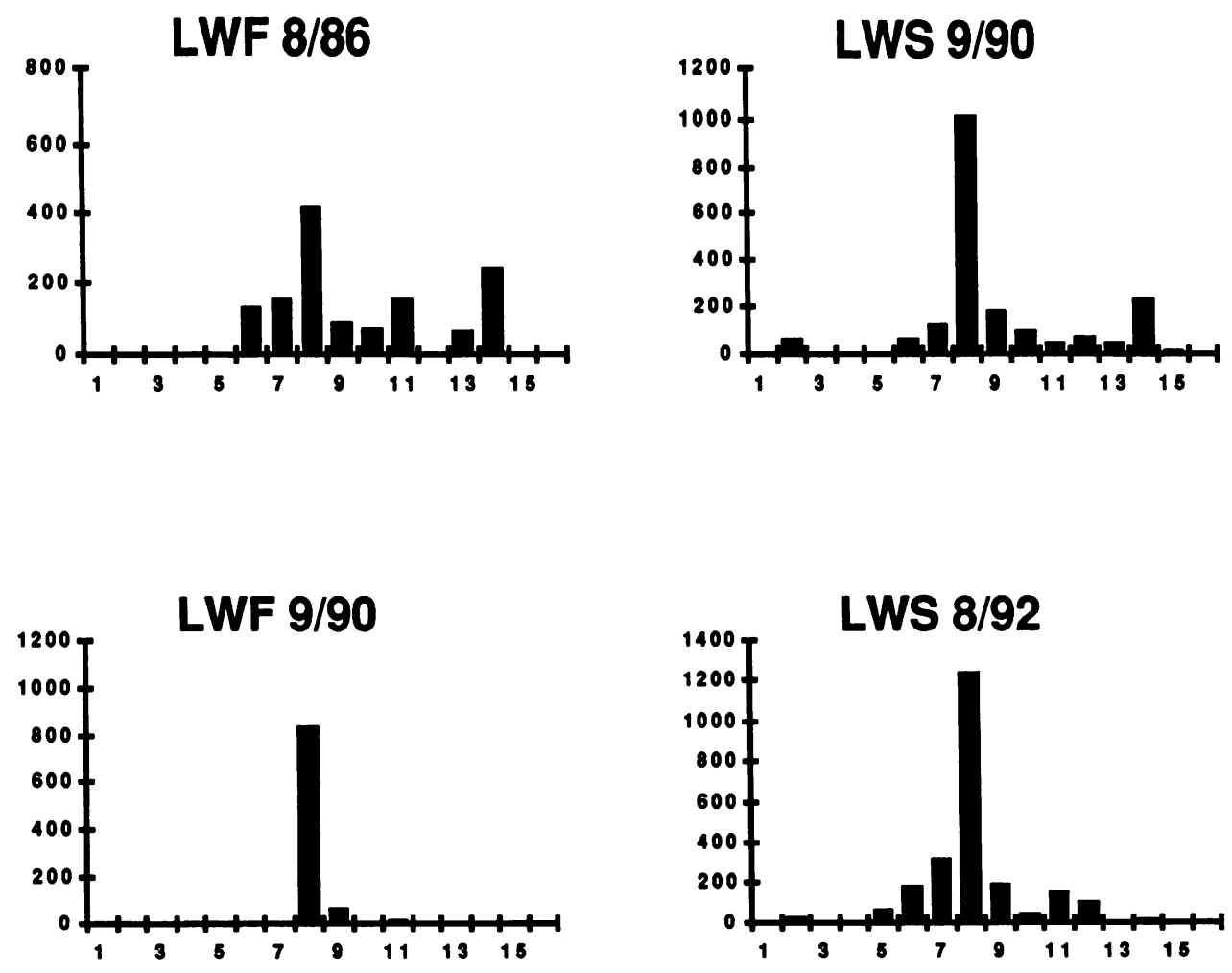

Figure 5. Fine specificity of antiV3 antibody. Epitope mapping was performed using Geysen peptides. Binding of antibody to each peptide is shown on the vertical axis as $A_{405} \times 10^{3}$ (minus binding to control pins with no peptide). The peptides attached to the supports are arrayed on the horizontal axis with the closest to the amino terminus on the left (1) and proceeding toward the carboxyl-terminal peptides to the right.

The peptides were

1 , VQLNQSVEINCT;

2, NQSVEINCTRPN;

3, VEINCTRPNNNT;

4, NCTRPNNNTRKS;

5, RPNNNTRKSIRI;

6, NNTRKSIRIQRG;

7, RKSIRIQRGPGR;

8, IRIQRGPGRAFV;

9, QRGPGRAFVTIG;

10, PGRAFVTIGKIG;

11, AFVTIGKIGNMR;

12, TIGKIGNMRQAH;

13, KIGNMRQAHCNI;

14, NMRQAHCNISRA;

15, QAHCNISRAKWN; and

16, CNISRAKWNNTL.

region, three different analyses were performed. The first was statistical and the others experimental. The statistical analysis was performed with the data from the Geysen peptide analyses. We compared the peptides that contained a mutation with the peptides that were bound by antibody and asked whether these were dependent events. We defined a peptide as a "valid" epitope if there was antibody binding (optical density $>0.2$ ) in more than one-half of the L.W.F. sera tested $(n=15)$. Using these criteria, there were five peptides representing an epitope, three of which contained mutations. In the total group of 283 peptides, the sites of the 18 mutations were represented in 61 peptides. The value for $\chi^{2}$ was 4.44 , indicating that the association of three mutations with the five epitopes was not because of chance $(P<0.05)$.

Two of the mutations occur in the immunodominant V1 and V3 loops. To test whether the mutations were induced by antibody selection, we constructed peptides that correspond to the original and mutated sequences. If the mutations were se- lected by antibody, then sera from the time before the mutation should bind to the peptide representing the original sequence, but not to the mutant. Results of the ELISA analysis are shown in Fig. 10. For both the V1 and the V3 loops, it is apparent that there was no difference in the binding of the antibody to the original or the mutant sequence at any point in time. These data suggest that antibody selection may not have been an important factor in the evolution of the mutations in the V1 and V3 loops.

Finally, we tested HIV isolates obtained from L.W.F. at different points in time to determine if they were susceptible to neutralization by serial autologous serum samples (Table I). The data reveal that between 1985 and 1987, coincident with the appearance of the mutations in the V1 and V3 loops, there was a change in the neutralization pattern of the virus. The 1985 isolate was neutralized by early sera ( 1986 and earlier) as well as by the IIIB typing serum; the 1987 isolates were neutralized by neither early sera nor the typing serum. Although this 

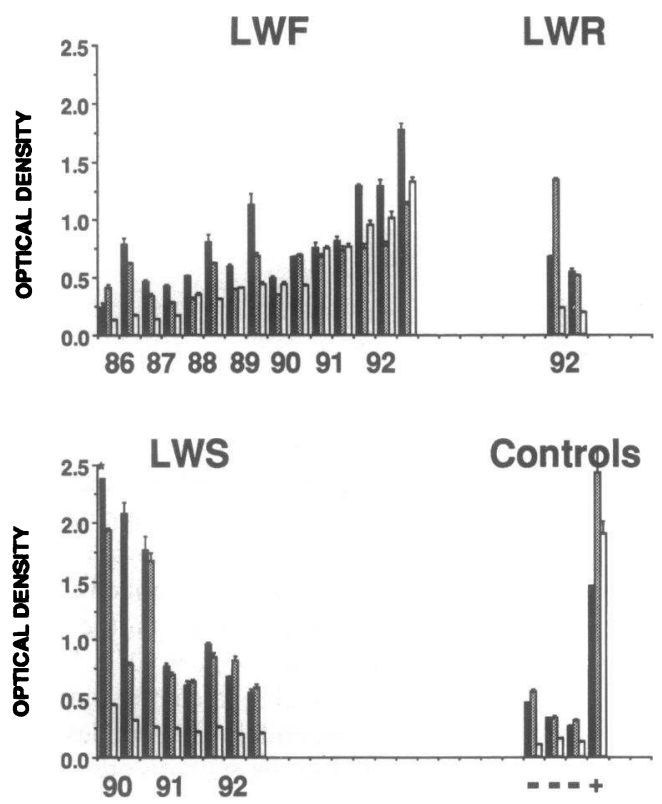

Figure 6. Binding of antibodies to heterologous V3 loop peptides. Crossreaction of laboratory worker sera to peptides representing the V3 loops of two heterologous strains (MN [dotted bars] and RF [white bars]) and the homologous strain (IIIB [black bars]) were tested by ELISA. Conditions were as described for Fig. 1.

may suggest that the later viruses arose as escape variants, it is noteworthy that the peak neutralization titer for each of these later isolates was present on the date that the isolates were obtained, indicating that the isolate existed in the presence of antibody capable of neutralizing it.
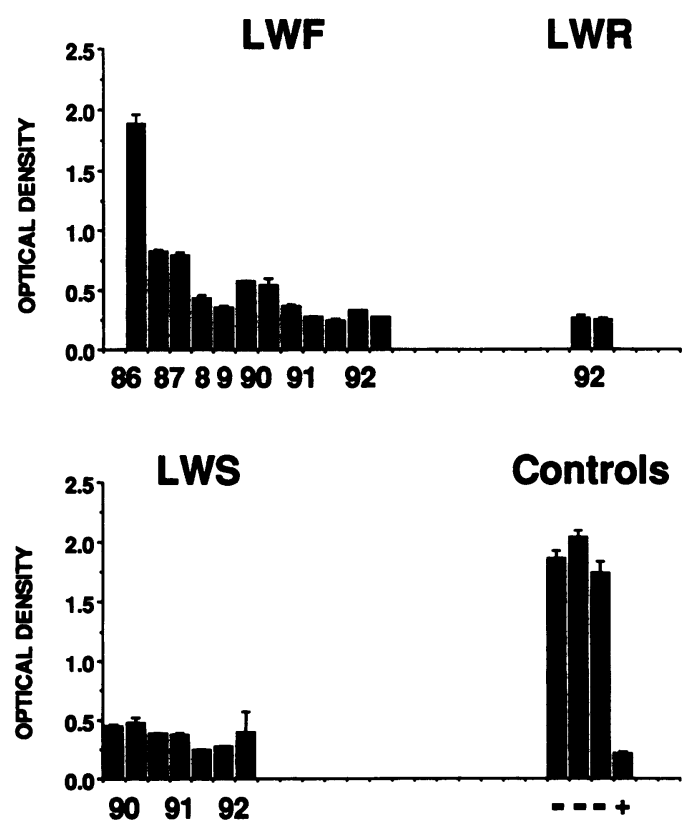

Figure 7. Inhibition of gp120/CD4 binding by laboratory worker sera. Inhibition of the interaction of gp120 and CD4 was measured by solid-phase ELISA in which gp 120 was immobilized, incubated with a 1:100 dilution of the test serum, and then with biotinylated CD4. The binding of CD4 was detected with avidin-alkaline phosphatase and $p$-nitrophenyl phosphate. Binding of CD4 to the solid phase is shown on the vertical axis as $A_{405}$. Thus, inhibition of binding is seen as a lower optical density. Results are mean and SD of triplicate samples.
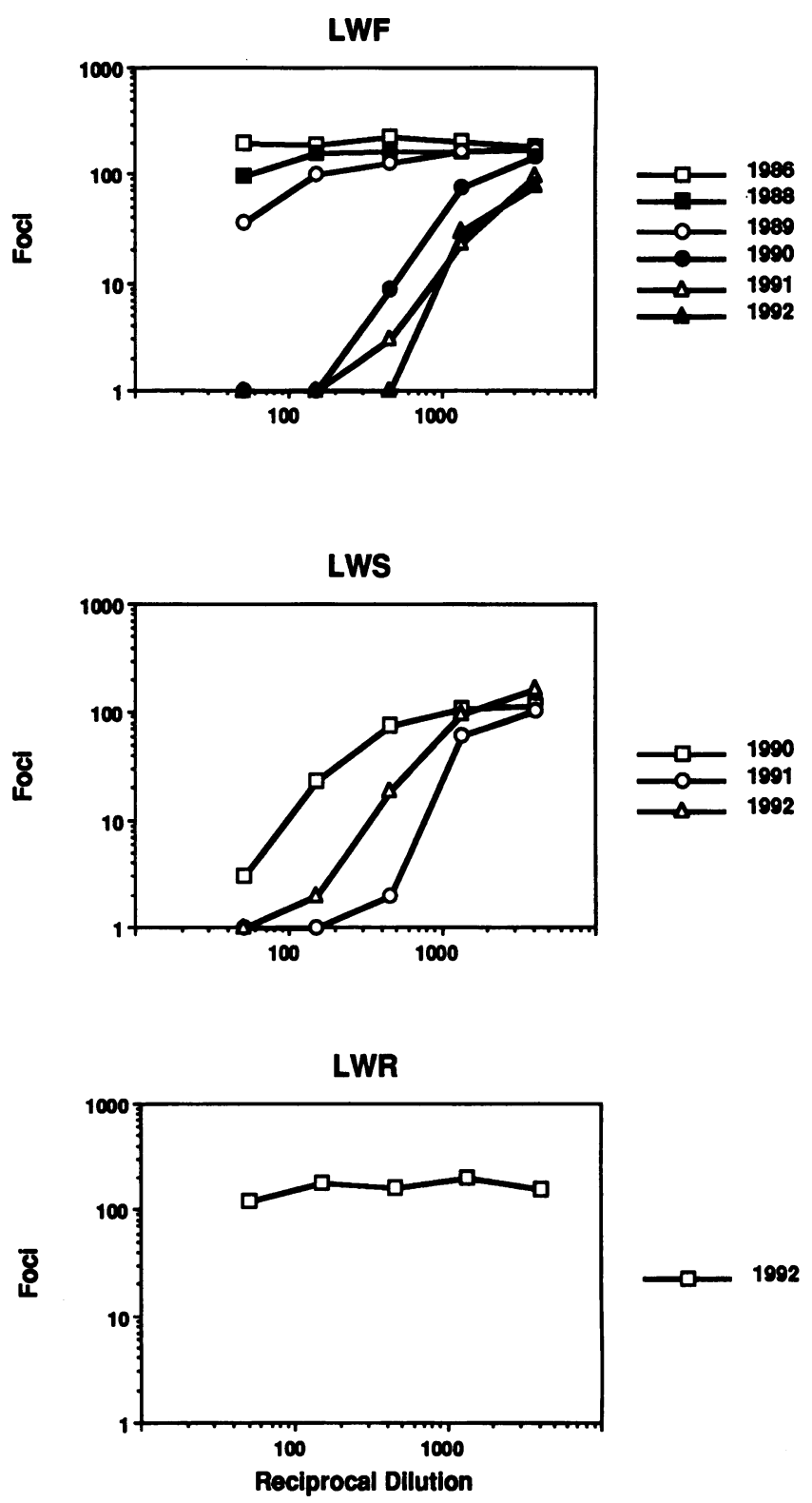

Figure 8 . Neutralization of homologous HIV by laboratory worker sera. Neutralization of the molecularly cloned HIV NL4-3 was measured by focal infectivity assay. Serum and virus preparations were incubated for $1.5 \mathrm{~h}$ and then transferred to a monolayer of CD4+ HeLa cells. The antibody-virus mixture remained on the monolayer for $3 \mathrm{~h}$ and was washed off, and the monolayer was incubated for 3 d. Foci were detected with anti-HIV sera and immunoperoxidase staining as well as by the characteristic morphology of multinucleated syncytia. The number of foci is shown on the vertical axis. Neutralization is seen as a diminution in the number of foci. Serum dilution is shown on the horizontal axis. Each point is the mean of duplicate samples. The number of foci in 18 control wells with normal human serum was $235 \pm 8$.

\section{Discussion}

In this paper we continue our analyses of the antibody response to the IIIB/LAV strain of HIV, which has included the HIV-infected laboratory workers, seronegative vaccinees in phase I clinical trials, and chimpanzees that have been vaccinated and then challenged with live $\operatorname{HIV}(3,4)$. By confining our studies 


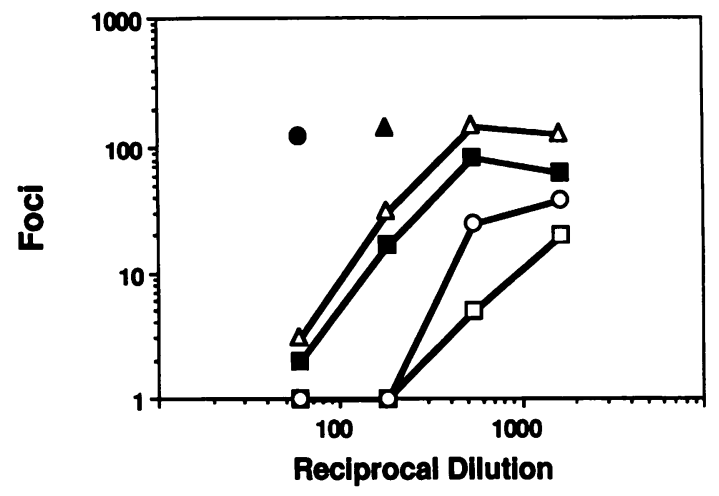

Figure 9. Neutralizing activity of anti-V1 antibody. Anti-V1 antibody was purified from L.W.F. serum on immobilized V1 peptide. Neutralizing antibody was detected using the focal infectivity assay as described in Fig. 8. The number of foci is shown on the vertical axis, and the antibody concentration is shown on the horizontal axis. Anti-V1 indicates the affinity-purified anti-V1 antibody from L.W.F. and anti-V3 is monoclonal antibody 924 . The initial concentrations of antibody before dilution were $1 \mathrm{mg} / \mathrm{ml}$. Start refers to the original serum before passage over the affinity column, while effluent is the serum after passage over the column (the dilution of the serum during column passage is corrected for in stating the reciprocal dilution). Each data point is the mean of duplicate samples.

to this group of individuals, we can study the antibody response on peptides, viral preparations, and recombinant proteins that correspond to the same strain of HIV to which the subjects have been exposed. Although there is some sequence microheterogeneity between different isolates of the IIIB/LAV strain,

\section{A V1 Loop}
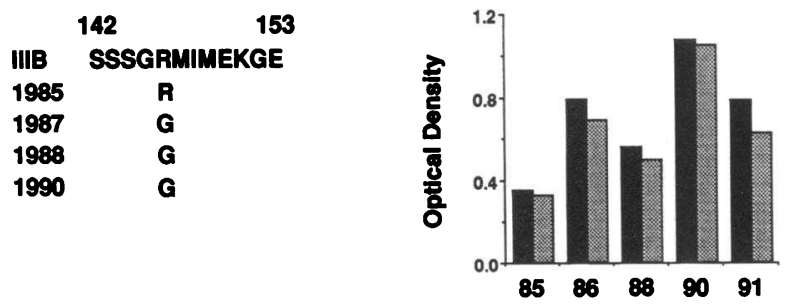

B V3 Loop

$\begin{array}{lcc} & 307 & 318 \\ \text { IIIB } & \text { IRIQRGPGRAFV } \\ 1985 & \text { A } \\ 1987 & T \\ 1988 & T \\ 1990 & T\end{array}$

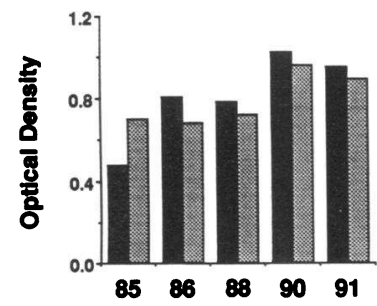

Figure 10. Binding of L.W.F. sera to peptides representing mutant sequences. Env sequences from L.W.F. have revealed mutations within the V1 loop $(A)$ and the V3 loop $(B)$. The original (black bars) and mutant (dotted bars) sequences are shown on the left side of each panel. Geysen peptides were constructed representing the original and the mutant sequence. Sera from the indicated dates were tested for binding to the peptides. Each point represents the mean of duplicate samples.
Table I. Neutralization of Serial HIV Isolates from L.W.F.

\begin{tabular}{lccccc}
\hline \multirow{2}{*}{$\begin{array}{c}\text { Date of } \\
\text { bleed }\end{array}$} & RF & IIIB & $9 / 4 / 85$ & $4 / 7 / 87$ & $9 / 30 / 87$ \\
\cline { 2 - 6 } & 4 & 4 & 4 & 4 & 4 \\
$9 / 4 / 85$ & 4 & $32-64$ & $8-16$ & 8 & 4 \\
$2 / 4 / 86$ & $8-16$ & $32-64$ & 8 & 4 & $4-8$ \\
$3 / 4 / 86$ & 4 & $8-16$ & $16-32$ & 4 & 4 \\
$8 / 5 / 86$ & 4 & 16 & $16-32$ & $8-16$ & 8 \\
$8 / 20 / 86$ & $8-16$ & $32-64$ & 16 & 16 & 4 \\
$9 / 16 / 86$ & 4 & $8-16$ & 16 & 4 & 4 \\
$10 / 15 / 86$ & $8-16$ & 128 & 32 & $16-32$ & $8-16$ \\
$2 / 11 / 87$ & 8 & $32-64$ & $32-64$ & $16-32$ & $16-32$ \\
$3 / 19 / 87$ & 8 & $32-64$ & 64 & $32-64$ & $8-16$ \\
$4 / 7 / 87$ & $32-64$ & $32-64$ & $64-128$ & $8-16$ & $16-32$ \\
$9 / 30 / 87$ & $32-64$ & $32-64$ & $64-128$ & $8-16$ & $16-32$ \\
$11 / 30 / 87$ & $4-8$ & $128-256$ & $64-128$ & $32-64$ & $16-32$ \\
$1 / 21 / 88$ & $16-32$ & $32-64$ & $32-64$ & $8-16$ & 16 \\
$5 / 4 / 88$ & $<4$ & 128 & 128 & 16 & 4 \\
IIIB typing sera & & & & & 4 \\
\hline
\end{tabular}

* Neutralization titer is the reciprocal of the dilution giving $90 \%$ inhibition of 150-200 synctium-forming units of virus.

we are able to epitope map the antibody response in far greater detail than has previously been possible.

We have shown previously that although vaccinated individuals make antibody to gp 160 , to the V3 loop, and to the CD4-binding site in titers comparable with infected individuals, there are significant differences in the quality of the antibody produced, particularly with regard to neutralization and fine specificity of the anti-V3 (3). We have also shown that immunization with vaccinia-gp 160 results in priming for an antibody response to a region of gp41 (AA 720-740) not normally immunogenic $(3,4)$. Here we follow the evolution of the antibody response with time in the three laboratory workers infected with HIV during the course of their work.

The IIIB/LAV strain of HIV has several unique characteristics that set it apart from other laboratory strains and clinical isolates of HIV. Most notable is the ability of this strain to cause a persistent infection of $T$ cell lines in which virtually all cells remain productively infected for indefinite periods of time (23). This characteristic appears to map to the envelope region $(23,24)$. Because of these unique characteristics as well as other differences between IIIB/LAV and clinical isolates, concern has been raised whether IIIB/LAV is a pathogenic form of HIV. Declining CD4 counts in all the infected laboratory workers and outright AIDS in one demonstrate that this strain of HIV can indeed cause disease despite its long in vitro passage. Moreover, the development of clinical features in the laboratory workers, in the absence of any other risk factors, clearly shows the Duesberg hypothesis, that HIV is not the cause of AIDS (25), to be untenable.

Perhaps the greatest surprise in these studies was the demonstration that the V1 loop of gp120 is both immunodominant and the target of neutralizing antibody. We have also found this to be true in chimpanzees challenged with IIIB/LAV (4). It is likely that this has been missed in previous studies because the V1 loop is hypervariable, and differences between the infecting isolate and the test strain did not allow for detection of antibody to this region $(1,2)$. An alternate explanation is that one of the unique characteristics exhibited by the IIIB/LAV 
strain is that the V1 loop has greater exposure than in clinical isolates. If these findings regarding the V1 loop can be confirmed in patients infected with more typical clinical isolates, then these findings have important implications for vaccine design. None of the current IIIB/LAV-based subunit vaccines induce antibody to the V1 loop, although in chimpanzees who received vaccinia-gp 160 and then were infected with HIV, antiV1 antibodies rose to higher levels after infection than in control chimpanzees $[(3,4)$ and Pincus, S. H., and K. G. Messer, unpublished data].

There are some important differences in the temporal evolution of the antibody response in the different laboratory workers. L.W.F., who was infected with an inapparent inoculum, had a slow rise in the level of all antibodies over the $7 \mathrm{yr}$ of follow-up. In contrast, L.W.S., infected with a large inoculum, had high levels of antibody from the initial time point. This may be a function of inoculum size, since similar, although less marked, findings have been seen in chimpanzees infected with a high versus low inocula (4). Another difference between L.W.F. and L.W.S. is the crossreaction of L.W.F. with the V3 loop of RF. It is possible that the differences between L.W.F. and L.W.S. are due to differences in the genetic makeup of the individuals affecting immune response genes, but with only two individuals this cannot be tested.

We can also draw correlations between clinical progression and the antibody response of the laboratory workers. L.W.F. has the least advanced clinical syndrome, with CD4 counts only recently beginning to drop. L.W.F. antibody levels have been climbing or on plateau levels throughout the observation period. L.W.S. has had a continually low and dropping CD4 during the postinfection period. The rapid progression of disease in this case may be related to inoculum size. During 1992 L.W.S. has had a fall in the levels of anti-gp160, anti-V3, and neutralizing antibody. This fall may be related to the advance in clinical disease. L.W.R., who has AIDS, has the lowest antibody levels of all of the laboratory workers, with background levels of neutralizing and anti-Gag antibodies. Unfortunately, only a few serum samples are available from this individual, so we cannot determine whether the fall in antibody levels preceded the development of AIDS.

In spite of the differences pointed out above, there were many consistencies in the anti-V3 response of all the laboratory workers, which were also seen in HIV-challenged chimpanzees (4). The most marked is that the tip of the V3 loop, which contains the conserved core sequence GPGR (16), is the immunogenic region of V3 (Fig. 5). This is in marked contrast to what is seen after vaccination, where other regions of the V3 loop are the antigenic sites [(3) and Pincus, S. H., and K. G. Messer, unpublished data]. The recognition of the core sequence as well as the crossreaction with peptides corresponding to other V3 loops suggest that the neutralization of nonhomologous strains of HIV by sera from infected individuals may in part be because of crossreactions of anti-V3 antibody (26).

The nature of neutralizing antibodies is important to assess. Generally it is believed that there are two components of neutralizing antibody: anti-V3 $(26,27)$ and CD4/gp1 20 blocking antibodies (2, 17-19). In previous studies we have shown that the titer of neutralizing antibodies correlates most closely with the fine specificity of anti-V3 antibodies and not with the titers of anti-gp160, anti-V3, or CD4/gp120 blocking antibody (3, 4). In this paper, we demonstrate that, at least in the laboratory workers, anti-V1 antibody appears to be an important compo- nent in neutralization. Anti-V1 antibodies do not block the interaction of CD4 and gp120 (data not shown). Also of interest is the finding that L.W.R. has very high levels of CD4/ gp120 blocking antibody, but no neutralizing activity. Because all of the sera tested have anti-V3 with the same fine specificity, we cannot correlate this with the titer of neutralizing antibody.

It has been suggested that the evolution of HIV during the course of infection in a single individual is the result of selection by antibody for escape variants (28). Because there have been complete Env sequences obtained from L.W.F. at multiple time points ${ }^{1}$ and we have mapped the antigenic regions, it was possible for us to test the hypothesis that antibody selects for mutant viruses. Our analyses yielded mixed results. The statistical association of epitopes and mutations as well as the fact that there were mutations in the V1 and V3 loops, both immunodominant sites, argue that antibody may indeed be selecting for the mutations. However, the experimental data, in which sera from times preceding the mutation bind peptides corresponding to the original and the mutant sequence equally, argue against antibody selection because the antibody would have selected equally against both the original and the mutant virus. Moreover, there were no mutations in regions of Env shown to result in escape mutants $(29,30)$ nor in any other neutralization epitopes. These results leave the relationship between mutation and antibody selection an open question. Similarly, our data on the neutralization of serial HIV isolates yielded conflicting information on this issue. There was clearly a shift in neutralization phenotype of the L.W.F. isolates between 1985 and 1987, suggestive of selection. However, the highest titer of neutralizing antibody to each of the later reisolates was present on the day the virus was cultured, indicating that the isolates could exist in the presence of neutralizing antibody.

The studies reported in this manuscript represent an unfortunate experimental opportunity. The risk factor that these laboratory workers shared in common was that all worked in facilities engaged in the preparation of highly concentrated batches of HIV. The infection of the laboratory workers with a defined strain of HIV has allowed us to evaluate in great detail the evolution of the systemic antibody response to HIV. Because these individuals are now receiving vaccine therapy (6), the natural evolution of the antibody response can no longer be studied. However, future studies will allow a detailed analysis of alterations induced by the therapeutic vaccine.

\section{Acknowledgments}

We admire the willingness of the anonymous laboratory workers who have been infected with HIV to allow themselves to be subjects of scientific investigation. However, no data derived from these important patients can serve to mitigate the personal tragedies involved. We thank John Swanson and Pat Fast for review of this manuscript and thoughtful suggestions. Carole Smaus and Susan Smaus provided excellent secretarial assistance.

Our work was supported by National Institute of Allergy and Infectious Disease and National Cancer Institute intramural research funds and the National Institutes of Health Intramural AIDS Targeted Anti-Viral Program.

\section{References}

1. Broliden, P.-A., A. von Gegerfelt, P. Clapham, J. Rosen, E.-M. Fenyo, B. Wahren, and K. Broliden. 1992. Identification of human neutralization-inducing regions of the human immunodeficiency virus type 1 envelope glycoproteins. Proc. Natl. Acad. Sci. USA. 89:461-465. 
2. Profy, A. T., P. A. Salinas, L. I. Eckler, N. M. Dunlop, P. L. Nara, and S. D. Puney. 1990. Epitopes recognized by the neutralizing antibodies of an HIV-1-infected individual. J. Immunol. 144:4641-4647.

3. Pincus, S. H., K. G. Messer, D. H. Schwartz, G. K. Lewis, B. S. Graham, W. A. Blattner, and G. Fisher. 1993. Differences in the antibody response to human immunodeficiency virus-1 envelope glycoprotein (gp160) in infected laboratory workers and vaccinees. J. Clin. Invest. 91:1987-1996.

4. Pincus, S. H., K. G. Messer, and S.-L. Hu. 1994. Effect of nonprotective vaccination on antibody response to subsequent human immunodeficiency virus infection. J. Clin. Invest. 93:140-146.

5. Weiss, S. H., J. J. Goedert, S. Gartner, M. Popovic, D. Waters, P. Markham, F. di Marzo Veronese, M. H. Gail, W. E. Barkley, J. Gibbons, et al. 1988. Risk of human immunodeficiency virus (HIV-1) infection among laboratory workers. Science (Wash. DC). 239:68-71.

6. Redfield, R. R., D. L. Birx, N. Ketter, E. Tramont, V. Polonis, C. Davis, J. F. Brundage, G. Smith, S. Johnson, A. Fowler, et al. 1991. A phase I evaluation of the safety and immunogenicity of vaccination with recombinant gp 160 in patients with early human immunodeficiency virus infection. $N$. Engl. J. Med. 324:1677-1684.

7. Myers, G., B. Korber, S. Wain-Hobson, R. F. Smith, and G. N. Pavlakis. 1993. Human Retroviruses and AIDS 1993. A Compilation and Analysis of Nucleic Acid and Amino Acid Sequences. Los Alamos National Laboratory, Los Alamos, NM.

8. Geysen, H. M., S. J. Rodda, T. J. Mason, G. Tribbick, and P. G. Schoofs. 1987. Strategies for epitope analysis using peptide synthesis. J. Immunol. Methods. 102:259-274.

9. Pincus, S. H., K. Wehrly, and B. Chesebro. 1991. Use of a focal infectivity assay for testing susceptibility of HIV to antiviral agents. BioTechniques. 10:336342.

10. Chesebro, B., and K. Wehrly. 1988. Development of a sensitive quantitative focal assay for human immunodeficiency virus infectivity. J. Virol. 62:37793788.

11. Chesebro, B., K. Wehrly, J. Metcalf, and D. E. Griffin. 1991. Use of a new CD4-positive HeLa cell clone for direct quantitation of infectious human immunodeficiency virus from blood cells of AIDS patients. J. Infect. Dis. 163:64-70.

12. Adachi, A., H. E. Gendelman, S. Koenig, T. Folks, R. Willey, A. Rabson, and M. A. Martin. 1986. Production of acquired immunodeficiency syndromeassociated retrovirus in human and nonhuman cells transfected with an infectious molecular clone. J. Virol. 59:284-291.

13. Lori, F., L. Hall, P. Lusso, M. Popovic, P. Markham, G. Franchini, and M. S. Reitz. 1992. Effect of reciprocal complementation of two HIV-1 molecular clones on HIV-1 cell tropism and virulence. J. Virol. 66:5553-5560.

14. Nara, P. L., and P. J. Fischinger. 1988. Quantitative infectivity microassay for HIV-1 and 2. Nature (Lond.). 331:469-470.

15. Javaherian, K., A. J. Langlois, C. McDanal, K. L. Ross, L. I. Eckler, C. L. Jellis, A. T. Profy, J. R. Rusche, D. P. Bolognesi, S. D. Putney, et al. 1989. Principal neutralizing domain of the human immunodeficiency virus type 1 envelope protein. Proc. Natl. Acad. Sci. USA. 86:6768-6772.

16. LaRosa, G. J., J. P. Davide, K. Weinhold, J. A. Waterbury, A. T. Profy, J. A. Lewis, A. J. Langlois, G. R. Dreesman, R. N. Boswell, and P. Shadduck.
1991. Conserved sequence and structural elements in the HIV-1 principal neutralizing domain. Science (Wash. DC). 249:932-935.

17. Steimer, K. S., C. J. Scandella, P. V. Skiles, and N. L. Haigwood. 1991. Neutralization of divergent HIV-1 isolates by conformation-dependent human antibodies to gp120. Science (Wash. DC). 254:105-108.

18. Back, N. K. T., C. Thiriart, A. Delers, C. Ramantarsing, C. Bruck, and J. Goudsmit. 1990. Association of antibodies blocking HIV gp120-sCD4 attachment with viral neutralizing activity in human sera. J. Med. Virol. 31:200-208.

19. Kang, C.-Y., P. L. Nara, S. Chamat, V. Caralli, T. Ryskamp, N. Haigwood, R. Newman, and H. Kohler. 1991. Evidence for non-V3-specific neutralizing antibodies that interfere with gp120/CD4 binding in HIV-infected humans. Proc. Nat. Acad. Sci. USA. 88:6171-6175.

20. Moore, J. P., and D. D. Ho. 1992. Antibodies to discontinuous or conformationally sensitive epitopes on the gp 120 glycoprotein of HIV-1 are highly prevalent in sera of infected humans. J. Virol. 67:863-875.

21. Lasky, L. A., G. Nakamura, D. H. Smith, C. Fennie, C. Shimasaki, E. Patzer, P. Berman, T. Gregory, and D. J. Capon. 1987. Delineation of a region of the human immunodeficiency virus type $1 \mathrm{gp} 120$ glycoprotein critical for interaction with the CD4 receptor. Cell. 50:975-985.

22. Sun, N.-C., D. D. Ho, C. R. Y. Sun, R. S. Liou, W. Gordon, M. S. C. Fung, X.-L. Li, R. C. Ting, T.-H. Lee, N. T. Chang, et al. 1989. Generation and characterization of monoclonal antibodies to the putative CD4-binding domain of human immunodeficiency virus type $1 \mathrm{gp} 120$. J. Virol. 63:3579-3585.

23. Pincus, S. H., R. L. Cole, E. M. Hersh, D. Lake, Y. Masuho, P. J. Durda, and J. McClure. 1991. In vitro efficacy of anti-HIV immunotoxins targeted by various antibodies to the envelope protein. J. Immunol. 146:4315-4324.

24. Pincus, S. H., and K. Wehrly. 1990. AZT demonstrates anti-HIV-1 activity in persistently infected cell lines: implications for combination chemotherapy and immunotherapy. J. Infect. Dis. 162:1233-1238.

25. Duesberg, P. H. 1991. AIDS epidemiology: inconsistencies with human immunodeficiency virus and with infectious disease. Proc. Natl. Acad. Sci. USA. 88:1575-1579.

26. Javaherian, K., A. J. Langlois, G. J. LaRosa, A. T. Profy, D. P. Bolognesi, W. C. Herlihy, S. D. Putney, and T. J. Matthews. 1990 . Broadly neutralizing antibodies elicited by the hypervariable neutralizing determinant of HIV-1. Science (Wash. DC). 250:1590-1593.

27. Goudsmit, J., C. Debouck, R. H. Meloen, L. Smit, M. Bakker, D. M. Asher, A. V. Wolff, C. J. Gibbs, Jr., and D. C. Gajdusek. 1988. Human immunodeficiency virus type 1 neutralization epitope with conserved architecture elicits early type-specific antibodies in experimentally infected chimpanzees. Proc. Natl. Acad. Sci. USA. 85:4478-4482.

28. Gegerfelt, A. V., J. Albert, L. Morfeldt-anson, K. Broliden, and E. M. Fenyo. 1991. Isolate-specific neutralizing antibodies in patients with progressive HIV-1-related disease. Virology. 185:162-168.

29. Reitz, M. S., Jr., C. Wilson, C. Naugle, R. C. Gallo, and M. Robert-Guroff. 1988. Generation of a neutralization-resistant variant of HIV-1 is due to selection for a point mutation in the envelope gene. Cell. 54:57-63.

30. Pincus, S. H., K. Wehrly, E. Tschachler, S. F. Hayes, R. S. Buller, and M. Reitz. 1990. Variants selected by treatment of human immunodeficiency virusinfected cells with an immunotoxin. J. Exp. Med. 172:745-757. 